Historic, Archive Document

Do not assume content reflects current scientific knowledge, policies, or practices. 

This price list cancels prices on ali Nursery Stock listed in our Spring 1919 catalog, and orders for this Fall delivery will be accepted only at prices named in this price list.

\section{Price List Fall 1919}

\section{Trees, Plants, Shrubs, Etc.}

\section{LEAMON G。TINGLE PITTSVILIE - MARYLAND}

My genera] catalog which illustrates, describes and prices all stock will be issued in January 1920. It will pay you to have a copy and I want you to have one and will send you one if you will fill out the enclosed post card and return to me at once.

TERMS. Cash with order; remit by money order, registered letter or draft.

SHIPPING SEASON. We begin filling orders about October 15th and continue digging and shipping during the entire Fall, Winter and Spring until May 10th. We are sometimes delayed in January and February by freezing weather but usually able to ship at times during these months.

NOTE. Except where noted 1 to 4 trees or plants are sold at the each rate; 5 to 49 at the 10 rate; 50 to 299 at the 100 rate; 300 to 599 at the 500 rate; 600 or more at the 1000 rate.

\section{Strawberry Plants}

We begin shipping strawberry plants abuot October 20 th and continue digging and shipping the entire fall, winter and spring to about May 10th. I expect to have more than twice as many as I have ever had any previous season but I believe there will be the biggest demand that has ever been known, so my advice is place your order for what plants you will want just as soon as you can. This advice applies to other stock as well.

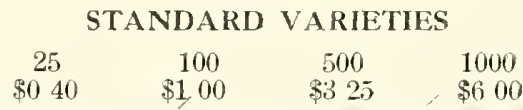

Amanda, Aroma, Bederwood, Big Joè, Brandywine Bubach, Big Valley, Champion K. Corsican. Campbell's Early. Dornan, Dr, Burrill. "Early Ozark. Early Jersey Giant. Ford, Glen Mary, Gandy! Hundred Dollar, Howard No. 17, Haverland, Hustler, Kellog's Premier, Kellog"s Prize, Kellogis Big Late, Klondyke Lady Thompson. Late Jersey Giant, Lupton, Missionary, Michel"s Early, Magic Gem. Marshall Messenger, McKinley, McAlpine, New York, Norwood, Parsons' Beauty, Pittsville, Ryckman, Rewastico, Sample, Success, Senator Dunlap, Sharpless, Tennessee* Wm. Belt and W Warfield.

$\begin{array}{cccc}25 & 100 & 500 & 1000 \\ \$ 060 & \$ 125 & \$ 500 & \$ 1000 \\ \text { CHESAPEAKE } & & \end{array}$

EVERBEARING VARIETIES

$\begin{array}{cccc}25 & 100 & 500 & 1000 \\ \$ 075 & \$ 200 & \$ 650 & \$ 1200\end{array}$

Americus. Ideal, Peerless, Progressive and Superb.

I have a small supply of Top Notch at $5 \mathrm{c}$ each.

If you will add 10 cents per 25 plants or 20 cents per 100 plants, I will send strawberry plants by mreel post, prepaid, to any part of Maine. New Hamphire, Vermont, Massachusetts, Connecticut. Rhode Island, New York, Ohio, Pennsylvania, New Jersey, Delaware, Maryland, Virginia, West Virginip

\section{Apple Trees \\ Each $10 \quad 100$ I yr. 3 to $4 \mathrm{ft} . \ldots \ldots \ldots \$ 045 \quad \$ 400 \quad \$ 3000$ $1 \mathrm{yr} .4$ to $5 \mathrm{ft} . \ldots \ldots \ldots \ldots \quad 60 \quad 550 \quad 4000$ $2 \mathrm{yr} .5$ to $6 \mathrm{ft} \ldots \ldots \ldots \ldots, 70 \quad 600 \quad 4500$ 4 yr. extra heavy ..... $100 \quad 900$}

\section{Partial list of varielies}

Baldwin, Wealthy, Grimes Golder, Jonathan, Staymens Winesap, York Imperial, Northern Spy, Yellow 'Transparent'- Early Harvest, Rome Beauty, $\llcorner$ Wolf River, LSpitzenburg, Hyslop (crab)

\section{Peach Trees}

\begin{tabular}{|c|c|c|}
\hline$y$ & $\begin{array}{l}\text { Each } \\
. \$ 050\end{array}$ & $\begin{array}{c}10 \\
\$ 400\end{array}$ \\
\hline 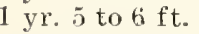 & .65 & 550 \\
\hline
\end{tabular}

\section{Partial list of varieties}

$\checkmark$ Elberta, Belle of Georgia, Greensboro, Hiley, White Heath Cling, Crawfords Early, Ray, $\checkmark$ Carman, Chairs I'hoice, J. H. Hale, Frances, Iron Mountain, Crawfords Late.

\section{Plum Trees}

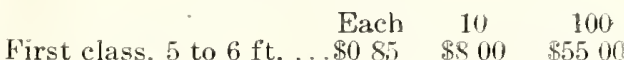
Varieties. Bradshaw, Burbank, Green Gage, German Prune, 'Abundance.

\section{Cherry Trees}

First class, 5 to $6 \mathrm{ft} \ldots$\begin{tabular}{ccc} 
Each & 10 & 100 \\
\hline 085 & $\$ 800$ & $\$ 5500$
\end{tabular} Varieties. Black Tartarian, Governor Wood, Early Richmond, English Morrello, Napoleon. Schmidts Bigarreau, Montmorency, Baldwin.

\section{Apricot Trees}

Firs $\tau$ class,$\ldots . . . \ldots . .75 \mathrm{c}$ each

Variely. Acme.

\section{Pear Trees} $\begin{array}{rrr}\text { Each } & 10 & 100 \\ 3 \text { to } 4 \mathrm{ft} . \ldots \$ 050 & \$ 400 & \$ 3000\end{array}$ $\begin{array}{llll}\text { First class, } 4 \text { to } 5 \mathrm{ft} \ldots & 60 & 550 & 4000 \\ \text { First class, } 5 \text { to } 6 \mathrm{ft} . \ldots & 70 & 600 & 4500\end{array}$

Varieties. Kieffer, Bartlett, Clapps Favorite, Seckel. 
Quince Trees

Each 10

First class.........\$0 $75 \quad \$ 650$

Varieties. Bourgeat, Champion.

\section{Grape Vines}

Strong Selected vines

$\begin{array}{llll}\text { Each } & 10 & 100 & 500\end{array}$

$\$ 020 \quad \$ 150 \quad \$ 1250 \quad \$ 5500$ 1000 Delaware, Empire State, Agawam, Brighton, Moores Early, Worden.

\section{Gooseberries}

$\begin{array}{ccc}\text { Varieties } & \text { Each } & 10 \\ \text { Downing, Josselyn. } & \$ 040 & \$ 300\end{array}$

\section{Currants}

$\begin{array}{ccc}\text { Varieties } & \text { Each } & 10 \\ \text { Wilder, Fays Prolific } & \$ 025 & \$ 200\end{array}$

\section{Raspberries}

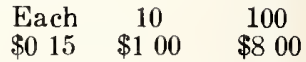

Varieties. St. Regis, Plum Farmer, Cuthbert.

\section{Blackberries}

Varieties

Each

$\$ 015$

$\begin{array}{cc}10 & 100 \\ \$ 100 & \$ 800\end{array}$

\section{Dewberries}

10 each

soc for 10

$\$ 700$ for 100

\section{Asparagus}

$\begin{array}{rrrr}10 & 100 \quad 500 & 1000\end{array}$

2 yr. No. $1 \ldots \ldots . \$ 040 \quad \$ 150 \quad \$ 400 \quad \$ 700$

1 yr. No. i.... $25 \quad 85 \quad 300 \quad 500$

Varieties. Giant Argenteuil, Barrs Mammoth,

Paldoetto, Conovers Collossal.

\section{Rhubarb}

3 yr. plants $10 \mathrm{c}$ each $75 \mathrm{c}$ for $10 \$ 500$ for 100

\section{California Privet}

$\begin{array}{rrrrr}10 & 100 & 500 & 1000\end{array}$

1 yr. 8 to 12 in. ....\$0 t0 $\$ 300 \$ 1300 \$ 2500$

1 yr. 12 to 18 in. ... 50 $3050 \quad 1600 \quad 3000$

1 yr. 18 to 24 in. .... $60 \quad 400 \quad 1800 \quad 3500$

2 yr. 18 to 24 in. ... $70 \quad 500 \quad 2100 \quad 4000$

2 yr. 24 to 36 in. ... $80 \quad 600 \quad 2600 \quad 5000$

\section{Barberry Thunbergii}

This is not the variety of Barberry which spreads wh at rust, and can safely be planted.

$$
\text { Each } \begin{array}{llll}
10 & 100 \quad 500 & 1000
\end{array}
$$

2 yr. 12 to 18 in. $\$ 015 \$ 100 \$ 800 \$ 3500 \$ 7000$ 3 yr. 18 to 24 in. $\quad 20 \quad 1501000 \quad 4500 \quad 9000$ 4 yr. 24 to $36 \mathrm{in} . \quad 25 \quad 2001200 \quad 500010000$

\section{Roses}

Two-year No. 1

Frau Karl Druschki, Margaret Dickson, Paul Neyron, Grus An Teplitz, Ked Baby Rambler, White Baby Rambler, Pink Baby Rambler, American Beauty \& $80 \mathrm{c}$.

White Dorothy Perkins, Crimson Rambler, Pink Dorothy Perkins, Blue Rambler ic 50c.

\section{Peonies}

Two-year, No. 1.

Red, White and Pink at $45 \mathrm{c}$ each.

Festiva Maxima at $60 \mathrm{c}$ each.

\section{Shade and Ornamental Trees}

Norway Maple 9 to $10 \mathrm{ft}$ Each $10 \quad 100$ $\begin{array}{lllllll}\text { Silver Maple } & 7 \text { to } 8 \mathrm{ft} . & 60 & 500 & 4500 \\ \text {.6 } & 8 \text { to } 9 \mathrm{ft} . & 75 & 700 & 6000\end{array}$ 9 to $10 \mathrm{ft} . \quad 95 \quad 900 \quad 8000$ 10 to $12 \mathrm{ft} . \quad 1 \quad 10 \quad 1000$
Schwedleri Maple 8 to $10 \mathrm{ft}, \quad 175 \quad 1700$ American Elm $\quad 10 \mathrm{ft}, 100 \quad 900$ Oriental Plane 8 to $10 \mathrm{ft} . \quad 150 \quad 1250$ Catalpa Bungeii $5 \mathrm{ft}$ ' stems 2 yr. heads 200 Catalpa Speciosa $\quad 10 \mathrm{ft} . \quad 75$ Horse Chestnut 6 to $8 \mathrm{ft}$. 100 Lombardy Poplar $\quad 10 \mathrm{ft} . \quad 125 \quad 110010000$

\section{Nut Trees}

American Sweet Chestnut 6-8 ft Each 10 7 to $8-8 \mathrm{ft} \$ 125 \$ 1000$ English Walnut $\quad 7$ to $8 \mathrm{ft} . \quad 150 \quad 1250$ $\begin{array}{llll}\text { Japan Walnut } & 5 \text { to } 6 \mathrm{ft} & 100 & 900 \\ \text { Butternut } & 6 & 75 & 700\end{array}$

$\begin{array}{llll}\text { Pecan } & 21 / 2 \text { to } 3 \mathrm{ft} . & 75 & 700\end{array}$

\section{Weeping Trees}

$\begin{array}{ccc}\text { Each } & 10 & 100\end{array}$ 8 to $9 \mathrm{ft} . \quad \$ 050 \quad \$ 400 \quad \$ 3000$ 9 to $10 \mathrm{ft} . \quad 60 \quad 500 \quad 4000$

Teas Weeping Mulberry

$6 \mathrm{ft}$. stems, $2 \mathrm{yr}$. heads $175 \quad 1600$

\section{Mulberries}

$\begin{array}{llrrr} & & \text { Each } & 10 & 100 \\ \text { Russian } & 4 \text { to } 5 \mathrm{ft} . & \$ 035 & \$ 300 & \$ 3000 \\ & 5 \text { to } 6 \mathrm{ft} . & 40 & 350 & 3500\end{array}$

\section{Evergreens}

\begin{tabular}{|c|c|c|}
\hline & & \\
\hline lorway Spruce 2 to $3 \mathrm{ft}$. & $\$ 100$ & $\$ 900$ \\
\hline 3 to $4 \mathrm{ft}$. & 125 & 1000 \\
\hline 4 to $5 \mathrm{ft}$. & 175 & 1600 \\
\hline 5 to $6 \mathrm{ft}$. & 225 & 2000 \\
\hline lorado Blue Spruce $1 \frac{1}{2}$ to $2 \mathrm{ft}$. & 100 & \\
\hline 3 to $4 \mathrm{ft}$. & 350 & \\
\hline ters Blue Spruce 2 to $21 / 2 \mathrm{ft}$. & 400 & \\
\hline aglas Spruce $11 / 2$ to $2 \mathrm{ft}$. & 60 & 550 \\
\hline 2 to $3 \mathrm{ft}$. & 100 & 900 \\
\hline to $4 \mathrm{ft}$. & 175 & 1600 \\
\hline merican Arborvitæ 2 to $3 \mathrm{ft}$. & 100 & 900 \\
\hline 3 to $4 \mathrm{ft}$. & 1 & 12 \\
\hline 4 to $5 \mathrm{ft}$. & 2 & 1750 \\
\hline oxwood, Pyramidal $21 / 2 \mathrm{ft}$. & 300 & \\
\hline ish Juniper 2 to $21 / 3 \mathrm{ft}$. & 100 & 900 \\
\hline hite Pine & 400 & \\
\hline $11 / 2$ to $2 \mathrm{ft}$. & 75 & 700 \\
\hline & & \\
\hline 5 to $6 \mathrm{f}$ & 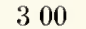 & \\
\hline opean Larch 5 to & 10 & \\
\hline $6 \mathrm{t}$ & 150 & \\
\hline
\end{tabular}

\section{Flowering Shrubs}

$\begin{array}{llll} & \text { Each } 10\end{array}$ Spirea Anthony Waterer 3 to $4 \mathrm{ft}$. $50 \quad 400$ Deutzia Crenata 3 to $4 \mathrm{ft}$. $\quad 50400$ 4 to $5 \mathrm{ft}$. $\quad 60500$

Deutzia Pride of Rochester 3-4 ft. $\quad 50 \quad 400$ Deutzia Gracillis 2 to $3 \mathrm{ft}$. $\quad 50400$ Deutzia Lemoine 2 to $3 \mathrm{ft}$. $\quad 50400$ Japan Blood Red Maple $3 \mathrm{ft} . \quad 300$

Hydrangea Paniculata Grandiflora 2 to $3 \mathrm{ft}$. $\quad 50400$ 3 to $4 \mathrm{ft}$. $\quad 75 \quad 650$

Hydrangea American Everblooming 2 to $3 \mathrm{ft}$. $\quad 50400$

White Snowball 2 to $3 \mathrm{ft} .50400$ Japan Snowball 2 to $3 \mathrm{ft}$. $60 \quad 500$ White Lilac 2 to $3 \mathrm{ft}$. $\quad 60500$ Charles X Lilac 2 to $3 \mathrm{ft}$. $\quad 60 \quad 500$ Mock Orange (Philadelphus) 2 to $3 \mathrm{ft}$. 50400 Weigela Eva Rathke 2 to $3 \mathrm{ft}$. 50400 Weigela Rosea 3 to $4 \mathrm{ft}$. $\quad 50400$ Rose of Sharon double r.d 3 to $4 \mathrm{ft} . \quad 50 \quad 400$ double white 3 to $4 \mathrm{ft} . \quad 50 \quad 400$ double blue 2 to $3 \mathrm{ft} \quad 40 \quad 300$ Forsythia Golden Bell 3 to $4 \mathrm{ft}$. $50 \quad 400$ Intermedia 2 to $3 \mathrm{ft} . \quad 40 \quad 300$ Butterfly Bush (Buddleia) 2 to $3 \mathrm{ft} . \quad 40300$ 3 to $4 \mathrm{ft} . \quad 50 \quad 400$ 4 to $5 \mathrm{ft} . \quad 75 \quad 6.50$ Silky Dogwood 3 to $4 \mathrm{ft}$. $50 \quad 400$ Scotch Broom 4 to $5 \mathrm{ft}$. $\quad 50400$ Wisteria Purple 4 to $5 \mathrm{ft}$. $\quad 50 \quad 400$ Wisteria White 4 to $5 \mathrm{ft}$. $\quad 50400$ Honeysuckle Halliana 4 to $5 \mathrm{ft}$. $\quad 50 \quad 400$ Pink Tartarian $2 \mathrm{ft}$. $\quad 50400$ 